\title{
Étude analytique de la formation initiale des enseignants de FLE selon le modèle TPACK
}

\author{
Dr. Nesrine Salah Abdel Ghany*
}

\section{Résumé}

Cette recherche vise à présenter une étude analytique de la formation initiale des futurs enseignants dans les Facultés de Pédagogie selon le modèle TPACK, l'un des modèles de l'intégration du numérique dans l'enseignement; la chercheuse a élaboré un questionnaire basé sur ce modèle afin de mesurer jusqu'à quel point les cursus enseignés par le biais du département de curricula et de méthodologie des facultés de pédagogie, reflètent-ils les axes de ce modèle qui tisse les connaissances pédagogiques et le contenu de la discipline visée avec la technologie, en vue d'intégrer le numérique d'une manière optimale et adaptée au niveau de nos apprenants et aussi aux circonstances spatiales de nos institutions; elle a accordé des rencontres avec le corps professoral pour évaluer comment les cursus enseignés sont dotés des compétences numériques du modèle TPACK, le calcul des résultats assure que ces cursus souffrent d'un manque crucial de ces compétences numériques qui ne dépassent pas le $20 \%$ de la totalité de ces cursus, ce qui a mené la chercheuse à présenter un cadre de formation suggéré qui aident les enseignants universitaires à intégrer les compétences numériques dans la formation initiale des futurs enseignants.

\section{Les mots clés :}

TICE- Le modèle TPACK - Intégration du numérique - Formation initiale - Cursus universitaire.

Tout système éducatif, et surtout le système éducatif égyptien a besoin de développer les compétences humaines et d'investir dans l'enseignement, pour que celui-ci devienne un instrument dynamique, se basant sur les besoins actuels et les évolutions futures, en vue de réduire l'analphabétisme, de construire et d'instruire le citoyen, de lui permettre de trouver un emploi - menant à terme à une réduction du chômage et une participation active au développement économique et social du pays.

Focalisons-nous un instant sur le système éducatif égyptien, qui a dû faire face à de nombreux défis durant des décennies, en proie à d'innombrables difficultés dans un monde où la production, les connaissances et le développement technologique se développent à toute vitesse, ce qui a eu un effet majeur sur la démographie du pays et sur le développement humain durable.

* Département de Curricula et de Méthodologie Faculté des Jeunes Filles pour les Lettres, les Sciences et la Pédagogie Université Ain Chams

Nesrine.salah@women.asu.edu.eg 
Ce qui a mené les pédagogues et les preneurs de décision à commencer la réforme attendue, en se focalisant sur le développement des enseignants, soit sur le niveau professionnel, académique et technologique, surtout, que les technologies de l'information et de la communication et de l'enseignement (TICE) sont devenues de plus en plus utilisées dans l'ensemble des domaines de l'activité humaine et elles exercent une influence grandissante sur le monde de l'éducation, et le débat sur l'efficacité des TICE pour l'apprentissage est encore d'actualité, les TICE font maintenant partie de l'environnement quotidien des étudiants, ainsi que du personnel enseignant.

Depuis la démocratisation de l'ordinateur, l'utilisation des technologies en éducation a suscité l'intérêt des chercheurs, de nombreuses recherches et rapports ont été publiés concernant le recours aux TICE pour soutenir l'enseignement et l'apprentissage. Plusieurs de ces travaux ont d'ailleurs contribué à l'avancement des connaissances dans ce champ. Les problématiques et les solutions pour résoudre le défi de l'intégration des TICE chez les enseignants sont bien connues. En effet, le rapport Horizon Report (Johnson, Adams Becker, Estrada et Freeman, 2015) mentionne que la formation initiale et continue des enseignants comportent des lacunes, qu'ils manquent de soutien lorsqu'ils rencontrent des obstacles, qu'ils n'ont pas perçu la valeur pédagogique des TICE ou qu'ils manquent de modèles pédagogiques. Ces problématiques ne datent pas d'hier, plusieurs de ces obstacles ont déjà été relevés dans la littérature scientifique, et ce, depuis quelques décennies (BECTA, 2003).

Depuis plusieurs années, le ministère de l'Enseignement et de l'Éducation en Égypte témoigne de l'importance de l'intégration des TICE au secondaire par la mise en place de mesures budgétaires pour l'achat de matériel informatique et pour la formation des enseignants.

Parallèlement, à cette évolution technologique, la réforme curriculaires devait être en cours, et des changements dans la formation initiale des enseignants doivent être préconisés.

Dans ce contexte, il est pertinent de se demander sur l'actualisation de la compétence professionnelle TICE des enseignants. Le questionnement, autrefois orienté vers le pourquoi de l'intégration des TICE, porte maintenant davantage sur le comment et le quoi de cette intégration. Il existe déjà certains référentiels de compétences TICE (Begin et al., 2000), mais ils demeurent très fortement centrés sur les compétences technologiques. Pourtant la capacité d'exploiter les TICE dans un contexte pédagogique doit maintenant faire partie des compétences de tout enseignant.

Comme le signale Perrenoud, $\mathrm{Ph}$. (1999), lorsqu'il a assuré que «ne rien dire des technologies nouvelles dans un référentiel de formation continue ou initiale serait indéfendable », il propose d'analyser les liens entre technologies d'une part, et opérations mentales, apprentissages et construction de compétences d'autre part. Ce qui mène les pédagogues à réviser les programmes de formation des enseignants selon cette nouvelle perspective, "une bonne école s'appuie effectivement sur de bons enseignants qui sont capables de mettre en pratique les développements pédagogiques les plus récents tant sur le plan du contenu de l'enseignement que 
de sa méthodologies »; ainsi, se baser sur la nouvelle technologie est devenue l'une des bases sur lesquelles s'est appuyé le développement de la formation des enseignants soit initiale ou continue.

Plusieurs efforts ont été assumés pour intégrer les TIC dans l'enseignement, et l'apparition du terme « techno pédagogie », pour désigner le domaine de l'utilisation pédagogique des technologies, demeure encore ambiguë. Alors plusieurs référentiels soumirent ne se veut pas neutre sur le plan des conceptions de l'enseignement et de l'apprentissage. Ils s'ancrent plutôt dans une conception socioconstructiviste de l'acte d'apprendre et participent ainsi à ce que Tardif (1998) définit comme le paradigme de l'apprentissage. "Savoir manipuler quelques outils ne suffit pas à garantir une bonne intégration des TIC dans l'enseignement. »

A ce stade, les pédagogues ont souligné l'importance et le besoin de se servir de modèles théoriques qui peuvent être utilisés comme schéma directeur au processus d'intégration des TICE dans toutes les dimensions, ainsi, un modèle peut avoir un ou plusieurs rôles en fonction de sa structure et de ses prescriptions. Il peut principalement : servir de canevas au processus d'intégration, en décrivant les différentes étapes à suivre et les tâches à effectuer pour mener à bien le processus, servir d'indicateur à l'état d'avancement du processus d'intégration dans une institution scolaire.

En se basant sur un modèle, on est donc à même de situer à quelle phase d'implantation des TICE on se trouve et ainsi évaluer les ressources nécessaires pour passer à la phase suivante, de décrire les différentes pratiques pédagogiques à employer dans un milieu scolaire pour se servir des TIC comme moyen d'enseignement. Quand même, le modèle permet de contextualiser des activités pédagogiques intégrant les TICE, et d'orienter la conception d'un curriculum de formation initiale et continue pour les enseignants, et il sert finalement à situer la place des technologies dans l'activité pédagogique en définissant les différents domaines pour lesquels son utilisation est optimale.

L'un de ces modèles est celui du TPACK (Technological Pedagogical And Content Knowledge), qui donne une représentation de l'intégration des technologies en classe. Il décrit comment les connaissances technologiques de l'enseignant s'articulent avec ses connaissances pédagogiques et didactiques pour une intégration réussie des technologies dans l'enseignement. Ce modèle décrit trois champs de connaissance de l'enseignant: la connaissance des contenus à enseigner, qui fait référence à la connaissance de la matière enseignée ; et qui dépend de la discipline et du degré scolaire (et qui représente dans cette recherche le FLE) la connaissance de la pédagogie qui corresponde à une connaissance approfondie des processus, des pratiques et des méthodes d'enseignement/apprentissage, enfin la connaissance de la technologie, qui fait référence, dans ce modèle, non seulement à la littératie numérique mais aussi à la maîtrise et à la compréhension en profondeur des technologies de l'information du point de vue du traitement de l'information, de la communication et de la résolution de problèmes. 
Afin de répondre aux exigences mondiales de l'intégration du TIC dans l'enseignement/apprentissage, il est temps de s'orienter vers la formation de l'enseignant qui représente le pivot de n'importe quelle réforme de l'éducation. Alors, peut-on se questionner « est ce que les programmes de formation initiale des enseignants dans les facultés de pédagogie suivent-ils l'un des modèles de l'intégration des TICE ?». Afin de répondre à cette question, la chercheuse a étudié les recherches récentes, elle a remarqué une rareté qui caractérise ce domaine ; parmi les études qui ont abordé ce thème, on cite celle de Faten Seoudy qui a effectué deux recherches qui se sont appuyées sur le modèle TPACK, dont le but de la première étude était l'évolution des programmes du développement professionnel des enseignants des sciences commerciales en 2017, tandis que la deuxième étude traite l'évolution des outils du stage pratique des futurs enseignants de la section des sciences en 2019. A l'instar de ces recherches, et par manque des études s'appuyant sur ce modèle surtout en didactique du FLE, la chercheuse élabore cette recherche dont le but principal est d'effectuer une étude analytique du programme de formation initiale des enseignants de FLE selon le modèle TPACK.

\section{Problématique de la recherche :}

La présente recherche vise à répondre à la question principale suivante : Comment analyser la formation initiale des futurs enseignants de FLE selon le modèle TPACK, l'un des modèles de l'intégration de la nouvelle technologie dans l'enseignement. De cette question émanent les questions suivantes :

- Quelles sont les performances technologiques et pédagogiques à intégrer dans les cursus de la formation initiale des futurs enseignants de FLE selon le modèle TPACK ?

- Dans quel mesure les performances technologiques et pédagogiques existent elles dans les cursus de la formation initiale selon le modèle TPACK ?

- Comment intégrer les performances technologiques et pédagogiques dans les cursus de la formation initiale des enseignants de FLE selon le modèle TPACK ?

\section{Objectifs de la recherche :}

L'objectif de cette recherche réside dans le fait de / d':

- Intégrer les performances technologiques dans la formation initiale des futurs enseignants de FLE en suivant le modèle TPACK.

- Concevoir un référentiel des performances technologiques et pédagogiques à intégrer dans les cursus de la formation initiale des futurs enseignants de FLE selon le modèle TPACK.

\section{Méthodologies de la recherche :}

La chercheuse a suivi la méthode descriptive /analytique pour accomplir cette recherche, elle a décrit le statut de la formation initiale des futurs enseignants en Égypte, elle a analysé les résultats de la recherche pour concevoir les performances technologiques et pédagogiques à 
intégrer dans les modules de la formation initiale des futurs enseignants de FLE selon le modèle TPACK

\section{Délimites de la recherche :}

Cette recherche se limite aux :

- Corps enseignants engagés dans la formation initiale des futurs enseignants et qui appartiennent au département de Curricula et de Méthodologie dans le Faculté de Jeune Filles pour les lettres, les sciences et la Pédagogie et qui se compose d'un professeur, un professeur adjoint, deux maitres de conférences, et une maitre assistante.

- Cursus du micro-enseignement enseigné aux étudiants de la deuxième année pédagogie durant une année scolaire.

- Cursus de la méthodologie française enseigné aux étudiants de la troisième année pédagogie durant une année scolaire.

- Cursus de la méthodologie française enseigné aux étudiants de la quatrième année pédagogie durant une année scolaire.

- Fiche du stage pratique accordée durant la troisième et la quatrième année de leurs études.

\section{Hypothèses de la recherche :}

On peut formuler les hypothèses de la recherche ainsi :

- Le cursus du micro-enseignement ne se conforme pas avec les axes du modèle TPACK.

- Le cursus de la méthodologie de la langue française étudié à la troisième année ne se convient pas avec les axes du modèle TPACK.

- Le cursus de la méthodologie de la langue française étudié à la quatrième année ne reflète pas les axes du modèle TPACK.

- La fiche du stage pratique ne réalise pas les axes du modèle TPACK.

\section{Procédures de la recherche :}

Pour aborder cette thématique, la chercheuse a suivi les étapes ci-dessous :

- Étudier le modèle TPACK.

- Choisir parmi les axes du modèle TPaCK ceux qui convient avec la formation initiale des futurs enseignants de FLE.

- Élaborer quatre grilles d'évaluation basées sur les quatre axes du modèle TPACK afin d'évaluer le programme de la formation initiale des futurs enseignants de FLE.

- Appliquer ces grilles sur les cursus de la formation initiale des futurs enseignants de FLE selon le modèle TPACK:(Le micro-enseignement, la méthodologie française de la troisième et la quatrième année et la fiches du stage pratique.) 
- Analyser les résultats de l'analyse de ces grilles.

- Proposer un cadre de formation basé sur le modèle TPACK et qui sert à évoluer la formation initiale des futurs enseignants de FLE.

\section{Importance de la recherche :}

On y présente l'importance de cette recherche pour les preneurs de décisions, le corps enseignant, les chercheurs et les futurs enseignants :

\section{En ce qui concerne les chercheures :}

- Préparer des grilles d'évaluation basées sur le modèle TPACK, servant comme références pour évoluer les programmes de formation pédagogique des futurs enseignants de FLE dans les facultés de Pédagogie.

- Ouvrir de nouvelles pistes devant les chercheurs et les concepteurs des manuels afin d'effectuer des recherches et de préparer des manuels reflétant ce modèle.

\section{En ce qui concerne les preneurs de décisions :}

- Attirer l'attention sur l'importance du modèle TPACK l'un des modèles de l'intégration du TIC dans l'enseignement.

- Évaluer le programme de la formation initiale des futurs enseignants de FLE selon le modèle TPACK, ce qui les aide à la réforme des facultés de Pédagogie.

- Orienter la conception d'un curriculum de formation initiale et continue pour les enseignants vers le modèle TPACK.

\section{En ce qui concerne le corps enseignant :}

- Proposer un cadre référentiel des compétences que les programmes de formation initiale des futurs-enseignants doivent refléter.

- Guider le travail pédagogique des formateurs d'enseignants à l'université pour évoluer leurs programmes en se basant sur ce modèle.

- Décrire les différentes pratiques pédagogiques à employer dans un milieu universitaire pour se servir des TIC dans l'enseignement.

- Se servir du modèle TPACK pour contextualiser des activités pédagogiques intégrant les TICE.

- Situer la place des technologies dans l'activité pédagogique en définissant les différents domaines pour lesquels son utilisation est optimale. 


\section{En ce qui concerne les futurs enseignants :}

- Aider les apprenants à intégrer les TICE ; tout en renforçant l'apprentissage tout au long de la vie, et promouvoir les valeurs de l'autonomie et du développement professionnel durable.

\section{Outils de la recherche :}

Pour accomplir cette recherche, la chercheuse a élaboré les outils suivants :

- Quatre grilles d'évaluation basées sur les axes du modèle TPACK afin d'évaluer le programme de la formation initiale des futurs enseignants.

- Cadre de formation proposé basé sur le modèle TPACK pour évoluer le programme de la formation initiale des futurs enseignants de FLE.

\section{Terminologie de la recherche :}

- Étude analytique : c'est une méthode utilisée dans le cadre d'études qualitatives, afin de développer un cas particulier, c'est une démarche didactique et pédagogique qui permet d'aborder certains points du programme par une approche originale. On vise par étude analytique dans cette recherche, la démarche d'analyse systémique qui permet de construire des notions et des raisonnements qui seront réinvestis afin d'intégrer le numérique dans les programmes de formation des enseignants dans les facultés de pédagogie.

- La formation initiale : c'est l'ensemble des cursus enseignés à la Faculté de pédagogie, dont quatre départements s'en chargent de cette formation: le département des fondements de l'éducation, le département de la psychologie, le département de la technologie de l'éducation et le département de curricula et de méthodologie. Ce dernier est responsable de l'enseignement des cursus sujets de la recherche actuelle et qui sont: «le micro-enseignement » étudié à la deuxième année et se déroule pendant un an, «la méthodologie française » enseigné durant la troisième et la quatrième année en fonction d'un semestre chaque année, et finalement le stage pratique qui aura lieu pour deux ans consécutifs : un jour par semaine, et qui débute le premier Octobre jusqu'au début d'avril, durant les deux années d'étude, et le stage pratique continu qui a lieu les deux dernières semaines du mois d'avril de la quatrième année de leurs études.

- Le modèle TPACK : c'est un acronyme anglais qui désigne «Technology, Pedagogy and Content Knowledge » et qui vise à articuler technologie, pédagogie et contenus disciplinaires. Cette articulation vise à mieux former les enseignants à l'utilisation raisonnée des TICE. Ce modèle sert à décrire les différents types de connaissances à acquérir par un enseignant afin d'intégrer les technologies dans ses pratiques éducatives. 


\section{Le cadre théorique :}

Pour aborder la thématique de cette recherche, la posture épistémologique se situe au croisement du champ de recherche didactique et du champ de recherche pédagogique, en ce qui concerne la réforme des curricula par le biais de l'intégration des TICE dans le système éducatif universitaire. C'est la raison pour laquelle, la chercheuse présente ci-dessus quelques outils conceptuels afin de toucher de près le domaine de la réforme des curricula tout en intégrant les TICE selon le modèle TPaCK.

Cette recherche est structurée selon une approche qui propose une découverte progressive du domaine de l'intégration du numérique à l'enseignement et son importance dans la réforme des curricula universitaires, en commençant par une description de la formation initiale et ses cursus.

La formation initiale : C'est la formation des étudiants qui envisagent de se lancer dans l'enseignement pédagogique et littéraire, ces étudiants débutent par l'études des matières académiques concernant la langue et la littérature française durant les deux premières années, ce qui les aident à avoir une bonne maîtrise de la langue ; les cursus concernant la didactique et la méthodologie débutent à la deuxième année de la formation et traitent des processus d'apprentissage, d'enseignement et de développement.

Ces cours qui résident dans le micro-enseignement, la méthodologie française et le stage pratique portent principalement sur des thèmes de didactique, ayant pour but de fournir aux futursenseignants les moyens qui leur permettent de travailler de façon indépendante sur le FLE, de leur apporter le soutien et les moyens nécessaires, et de leur présenter des méthodes de mise en œuvre de cet enseignement dans le cadre de leurs cours.

Ce programme de formation a pour but de concevoir les principes de l'enseignement du FLE en précisant le profil d'un enseignant efficace; d'entraîner les étudiants à préparer le cours en utilisant les documents authentiques et en élaborant des activités langagières, et de les sensibiliser aux techniques de questionnement afin de pratiquer au maximum les procédures d'évaluation tout en variant les styles d'apprentissage, les entrainer aux techniques de l'auto-apprentissage en utilisant l'Internet dans la préparation des cours ; et de les préparer à l'enseignement de la lecture, la grammaire et la phonétique en vue de développer leurs compétences linguistiques et pédagogiques.

Mais la partie concernant l'Internet et son utilisation demeure une partie théorique qui ne se reflète ni dans la planification des leçons durant leurs stages pratiques, ni dans les travaux d'années visés accomplir durant leurs études.

\section{L'intégration du numérique dans l'enseignement du FLE :}

Dès qu'on désigne le numérique dans l'éducation, il est question de son intégration. Dans le dictionnaire Trésor de la langue française, le terme intégration est défini comme "l'action d'incorporer un ou plusieurs éléments étrangers à un ensemble constitué, d'assembler des éléments divers afin d'en constituer un tout organique; passage d'un état diffus à un état 
constant, résultat de l'action ». Autrement dit, l'intégration est un processus, c'est une suite d'opérations, d'ajustements, d'aménagements successifs.

Dans les discours sur le numérique, qu'ils soient scientifiques, institutionnels ou pédagogiques, il est indispensable de distinguer différents usages du numérique éducatif. Bien que les catégories ne se recoupent pas toujours et que leurs dénominations fluctuent, il faut distinguer entre les trois cas suivants : (avec, au et dans), former avec le numérique, former au numérique, former dans le numérique. Afin de préciser dans quelle mesure ces trois perspectives peuvent aider à comprendre les enjeux du numérique.

a. Former avec le numérique : désigne que le numérique est considéré comme un moyen, un outil, au service du développement des compétences en langue. Comme le cas d'utiliser le «WhatsApp », pour encourager les échanges en langue cible au sein de la classe, aussi bien à l'écrit qu'à l'oral, dans une modalité asynchrone ; et aussi l'utilisation d'autres logiciels comme «Audacity » (C'est un logiciel qui permet d'enregistrer, de jouer, d'importer et d'exporter des données en plusieurs formats dont WAV, AIFF et MP3. On peut traiter des sons avec les commandes Couper, Copier et Coller (avec annulations illimitées), combiner les pistes et ajouter des effets aux enregistrements.) pour concevoir des documents sonores, les cartes postales, les audioguides ou les journaux de bord; et d'autres logiciels comme Powtoon, ToonDoo, etc., pour des productions multimodales.

b. Former au numérique est considéré comme un objet, selon (Fastrez et De Smedt 2013), il s'agit, d'apprendre aux étudiants à "évoluer de façon critique et créative, autonome et socialisée dans l'environnement médiatique contemporain ». Il s'agit de faire apprendre des compétences telles que: savoir chercher, évaluer, organiser l'information; savoir communiquer et collaborer en utilisant différents objets techniques ; savoir gérer et diffuser des contenus; et enfin savoir se protéger des dangers de l'Internet et développer un esprit critique.

Bref, former au numérique, c'est former aux aspects techniques.

c. Former dans le numérique est considéré comme un milieu dans lequel se déroule tout ou partie d'un grand nombre de pratiques sociales: vendre, acheter, participer à des actions citoyennes, préparer un voyage, entretenir et nouer des relations, chercher un emploi, etc.

Le tout à partir d'ordinateurs fixes des tablettes ou des téléphones intelligents. Une activité, commencée en présentiel, peut se poursuivre à distance, ou inversement. Amener les apprenants à prendre part à des situations de communications authentiques en ligne est susceptible d'amener plusieurs changements.

Alors, le numérique est un objet multiple, la simple utilisation de tel ou tel objet technique en classe, ne suffit pas en soi pour que l'on puisse parler d'intégration et il faut s'assurer qu'il y ait une réelle valeur ajoutée sur le plan des apprentissages, et que les 
spécificités des objets techniques en question soient prises en considération, et enfin que l'expérience même d'enseignement et d'apprentissage soit modifiée.

Pour intégrer le numérique dans l'enseignement, tant de modèle ont apparue à ce stade, ce qui parait utile de présenter dans les lignes suivantes, les modèles de l'intégration des TICE qui ont mené à l'émergence du modèle TPACK ; (Sylviane BACHY 2014) dans sa recherche, a proposé un modèle théorique intégrateur servant à comprendre les différentes influences qui déterminent les pratiques pédagogiques des enseignants. Elle a présenté un outil permettant de dresser des profils d'enseignants en ce qui concerne l'influence de leur culture disciplinaire, de leur épistémologie personnelle, de leurs connaissances pédagogiques et de leurs connaissances technologiques.

(Mathieu THIBAULT 2017) dans sa thèse de doctorat a déterminé les enjeux à considérer pour la formation à l'enseignement des probabilités du secondaire avec des outils technologiques, il a décrit les enjeux liés aux connaissances et aux compétences à partir des modèles : (TPACK) ; et (Tapan) et il a décrit les enjeux liés à chacun d'eux. Les résultats de ces recherches ont aidé les enseignants et les chercheures souhaitant se lancer dans ce genre d'activité innovante; et ci-dessous, la chercheuse présente les modèles qui précèdent le modèle TPACK et qui ont aidé à son émergence.

Le modèle (PCK : ce terme se traduit en français par les connaissances pédagogiques du contenu, (Sensevy, 2009) affirme qu' «on peut le définir comme la connaissance que l'on a d'un savoir à des fins d'enseignement.» Il s'agit d'une forme de savoir pratique utilisée par les enseignants pour guider leurs actions. Pour (Shulman 2004) ce savoir comprend

- une compréhension de la façon de structurer et de présenter le sujet à apprendre ;

- une compréhension des conceptions communes des difficultés que rencontrent les apprenants lors de l'apprentissage d'un sujet particulier ;

- une connaissance des stratégies d'enseignement qui sont efficaces pour répondre aux besoins d'apprentissage des étudiants dans des circonstances particulières en classe.

L'enseignement dépend de l'organisation de systèmes des connaissances qui touchent à l'apprentissage et à l'enseignement et qui touchent au contenu.

Les connaissances du contenu comprennent les connaissances qui portent sur la matière qui doit être apprise ou enseignée. Les enseignants doivent avoir la connaissance des sujets qu'ils enseignent incluant les faits majeurs, les concepts, les théories et les procédures dans leur champ disciplinaire.

Les connaissances pédagogiques comprennent les connaissances sur les méthodes d'enseignement et d'apprentissage, la manière de gérer la classe, les évaluations, etc. «L'enseignant qui a de bonnes connaissances pédagogiques comprend comment les étudiants construisent leurs connaissances, acquièrent des compétences et développent de bonnes habitudes au regard de l'apprentissage » (Mishra \& Koehler, 2006). 
Selon (Shulman 2007) avoir la connaissance du sujet traité et des stratégies pédagogiques générales n'est pas suffisant pour qualifier de bons enseignants. Les connaissances pédagogiques du contenu (PCK) rendent mieux compte de la manière dont certains contenus devraient être enseignés. Selon Mishra et Koehler (2006) le cœur du PCK est la manière de transformer l'objet pour l'enseigner.

Le PCK propose d'expliquer les pratiques des enseignants. Il combine le contenu et la pédagogie pour comprendre comment certaines thématiques sont représentées et adaptées pour qu'elles soient apprises. Il s'agit d'étendre les connaissances du savoir en connaissance pour enseigner le savoir.

(Shulman 2007) a ajouté aux connaissances du contenu et aux connaissances pédagogiques : le savoir sur le curriculum (issu des programmes), le savoir concernant les apprenants, le savoir ayant trait aux contextes éducatifs, le savoir portant sur les finalités éducatives et le savoir propre de la profession.

Pour le niveau universitaire, (Lenze1995) a transformé le PCK en Discipline specific Pedagogical Knowledge (DPK) afin de mieux tenir compte des particularités de ce niveau d'enseignement à l'université, elle assure que le contenu enseigné par des professeurs d'université correspond en fait aux disciplines académiques en tant que telles. Dès lors, contenu et discipline sont envisagés comme des termes équivalents. L'adaptation du modèle de (Shulman 1986) pour le public universitaire a renforcé l'idée qu'il existe des spécificités disciplinaires qui influencent les connaissances pédagogiques des enseignants de ce niveau et que celles-ci ne peuvent pas être ignorées si l'on souhaite comprendre les pratiques d'enseignement.

Ces études ont mené aussi à l'émergence du modèle TPACK traité ci-dessous.

\section{Le modèle TPACK :}

Ce modèle développé par les professeurs titulaires Punya Mishra et Matthew J. Koehler (Koehler et Mishra 2009, Mishra \& Koehler 2006) a été inspiré du modèle PCK de Lee Shulman (Shulman, 1986) qui est basé sur le concept de « pédagogie du contenu disciplinaire ».

TPACK ajoute à PCK la « dimension technologique » et de ce fait décrit «l'exploitation optimale de la connaissance technologique dans les pratiques pédagogiques et le contenu disciplinaire ». Il est basé sur trois composantes fondamentales qui sont : la technologie, la pédagogie et le contenu. Il met en évidence les relations qui existent entre ces trois composantes (formant ainsi des composantes dérivées, et constitue ainsi une base théorique au curriculum de formation initiale et continue intégrant les TICE que doivent suivre les enseignants.

- La connaissance technologique (Technological Knowledge:TK) fait référence à la culture et à l'utilisation des nouvelles technologies comme Internet, la vidéo numérique, les ordinateurs, les IPhones, IPad, etc. Elle intègre la connaissance des notions de systèmes d'exploitation, la création d'archives, la création de documents et de diapositives, l'environnement matériel de l'ordinateur et ses périphériques, l'utilisation des logiciels et 
du web. Les enseignants doivent donc toujours être à jour sur les innovations des TICE pour pouvoir de mieux en mieux s'en servir dans leur environnement au profit de l'éducation.

- La connaissance pédagogique (Pedagogical Knowledge: PK) fait référence à la connaissance des modèles, des stratégies, des techniques et des méthodes d'enseignement /apprentissages permettant d'atteindre un but éducatif en instance. Elle est liée aux différentes perspectives de l'apprentissage des étudiants, à la gestion de la classe, à la mise en œuvre et la dispense des leçons ainsi qu'aux techniques d'évaluation. Un enseignant ayant des connaissances avancées en pédagogie comprend les mécanismes d'enseignement / apprentissage qui permettent d'avoir un rendu optimal pour ses étudiants, il effectue des bons choix au niveau des méthodes pédagogiques à utiliser pour l'atteinte de ses objectifs, il effectue une bonne gestion de la salle de classe, communique avec les étudiants et leurs parents. Cette connaissance inclut aussi la maîtrise des différentes théories de l'apprentissage et des procédés de psychopédagogie en vue d'une mise en pratique en situation réelle d'enseignement /apprentissage.

- La connaissance du contenu (Content Knowledge : CK) fait référence à la connaissance de l'objet d'enseignement/apprentissage réel. Le contenu est spécifique à une discipline ou un domaine bien précis de l'éducation. Comme c'est le cas de la langue française dans notre recherche proposée. L'enseignant doit maîtriser le contenu de la discipline qu'il enseigne, il doit en connaître toutes les bases, les théories, les concepts et l'idéologie, comprendre quelles sont les différentes relations qui s'appliquent entre les modules de sa discipline et entre sa discipline et les autres, et savoir les expliciter, les enseignants qui n'ont pas cette intelligence peuvent dénaturer l'objet de leur enseignement envers leurs étudiants.

- La connaissance de la pédagogie du contenu (Pedagogical Content Knowledge (PCK) : fait référence à un amalgame entre la pédagogie et le contenu disciplinaire. (Shulman 2007) a fait valoir le fait qu'avoir la connaissance disciplinaire et des stratégies pédagogiques générales, bien que nécessaire, ne sont pas suffisantes pour un enseignement de qualité, il a proposé une approche qui examine la relation entre ces deux concepts en introduisant la notion de PCK qui s'intéresse à :

- la représentation et à la formulation des concepts, des techniques pédagogiques,

- la connaissance de ce qui rend les concepts difficiles ou faciles à apprendre,

- la connaissance des connaissances antérieures des étudiants et des théories de l'épistémologie.

- Elle implique également la connaissance des stratégies d'enseignement qui intègrent des représentations conceptuelles appropriées afin de favoriser de manière significative la compréhension.

- Il comprend également la connaissance de ce que les étudiants apportent à la situation d'apprentissage. 
PCK représente le mélange du contenu et de la pédagogie dans la compréhension de la façon dont les aspects particuliers des objets sont organisés, adaptés et représentés pour l'enseignement.

- La connaissance de la Technologie liée au contenu (Technological Content Knowledge : TCK) fait référence à la manière dont la connaissance de la technologie (CT) et la connaissance du contenu disciplinaire $(\mathrm{CK})$ sont réciproquement liées l'une à l'autre. L'enseignant aspirant à cette connaissance doit non seulement maîtriser le contenu disciplinaire de la matière qu'il enseigne, mais doit aussi avoir connaissance des nouvelles technologies et savoir comment les applications liées à ces dernières peuvent modifier ou améliorer certaines notions du contenu disciplinaire et inversement comment le contenu disciplinaire en question peut participer à l'amélioration de la technologie.

- La connaissance de la Technologie liée à la pédagogie (Technological Pedagogical Knowledge : TPK) fait référence au lien existant entre la connaissance de la technologie (CT) et la connaissance de la pédagogie (PK). Elle permet d'établir l'impact que la technologie a sur la pédagogie et vice-versa. Elle est la connaissance de l'existence des différentes technologies et de leurs capacités à améliorer une situation d'enseignement

lapprentissage.

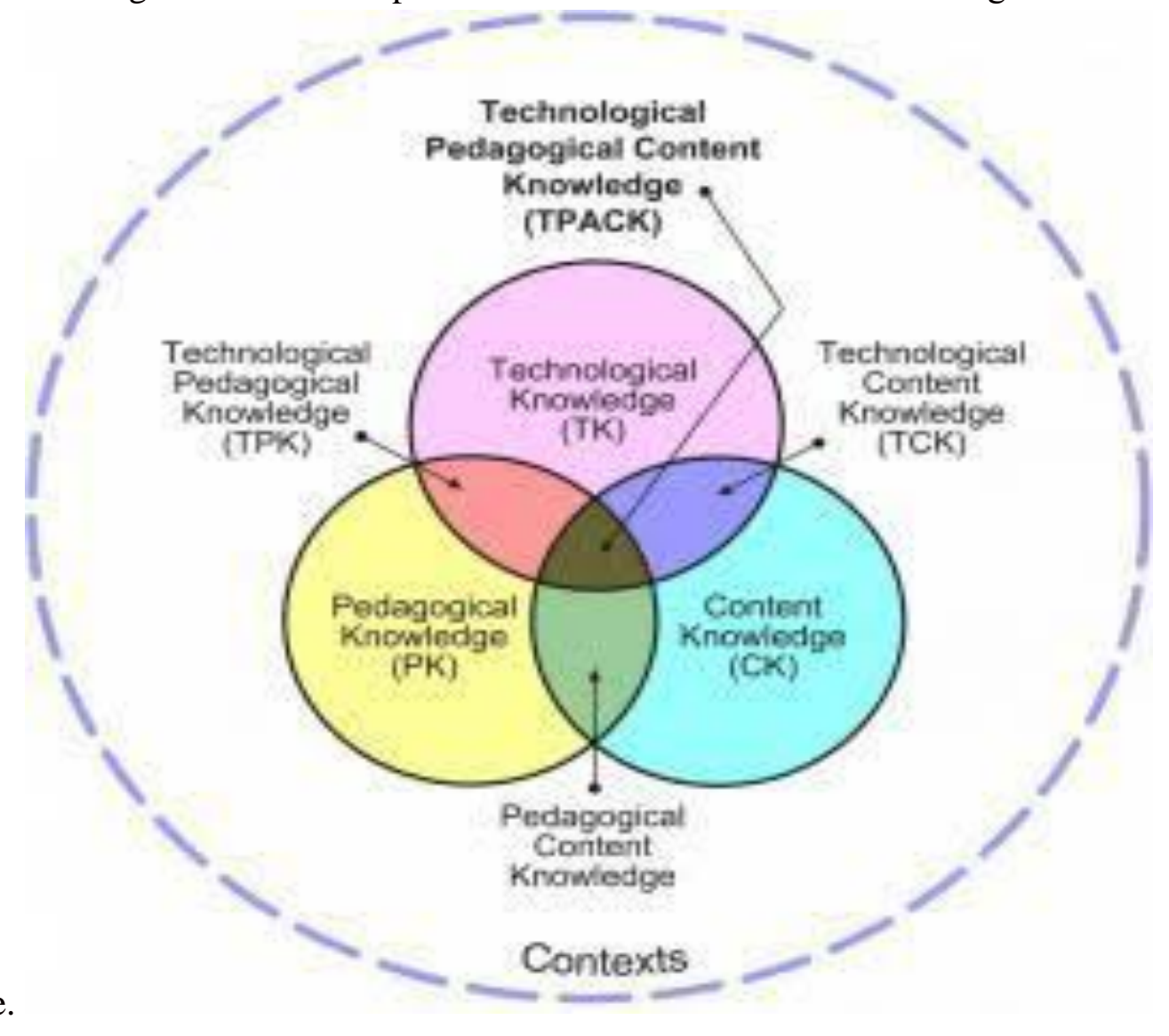

Modèle TPACK (Koehler et Mishra. 2009) traduit en français (Lefebre.2014) 
Après cette présentation du modèle TPACK et les modèles qui ont accéléré son émergence, on peut assurer que c'est un modèle qui défend l'idée d'interrelations fortes entre contenu, pédagogie et technologie, intégrant le contenu dans une dynamique à double sens, de même l'apparition des technologies en ligne force les enseignants à trouver un équilibre entre ces trois dimensions souvent les conduisant à se questionner à propos de leur pédagogie surtout que les programmes actuels de formation des enseignants ne leur fournissent pas les expériences nécessaires pour qu'ils puissent utiliser les technologies, ce qui mené la chercheuse à présenter ci-dessous le cadre pratique concernant la manière dont la chercheuse à adopter afin d'évoluer le programme de formation initiale en se basant sur les axes composants ce modèle.

\section{Le cadre pratique}

Pour pouvoir mener son étude analytique de la formation initiale des enseignants du FLE selon le modèle TPACK, la chercheuse suit les procédures suivantes :

1- L'élaboration des grilles d'évaluation de la formation initiale basée sur le modèle TPACK (Annexe1):

La chercheuse élabore quatre grilles d'évaluation qui valorisent les quatre axes choisis et adaptés selon les besoins et la nature des variables de la recherche, le but de ces grilles est de déterminer jusqu'à quel point les cursus du (micro-enseignement, de la méthodologie de la troisième et la quatrième année et les fichiers de la stage pratique) reflètent-ils les performances de l'intégration du numérique inclues dans le modèle TPACK, chaque grille présente un axe du modèle.

\section{Description :}

- La première grille intitulée la connaissance pédagogique : se compose de 15 performances reflétant la capabilité de l'étudiant à organiser les sujets et les concepts traités, d'une façon cohérente et intégrée et à varier les situations d'enseignement/apprentissage basées sur les théories pédagogiques; à gérer la classe et le comportement de ces étudiants tout en tenant compte de les guider et de les orienter vers les nouveaux savoirs en se basant sur les théories variées de l'enseignement.

- La deuxième traitant la technologie et le contenu : est composée de 15 performances cloisonnant les compétences de manipulation du contenu enseigné avec celles de l'intégration du numérique d'une manière adéquate et appropriée, telles que le choix et la variation des outils technologiques qui débouchent sur les objectifs escomptés, tout en mettant en compte le résout des problèmes liés aux diapositives utilisées, aussi les compétences reliées à l'utilisation optimale des nouveautés du Web tout en profitant des accès Internet qu'offrent leurs institutions, et l'utilisation des logiciels spécialisés dans les évaluations adaptées aux contenus, et aux niveaux des apprenants.

- La troisième concernant la pédagogie et le contenu se compose de 10 performances qui visent à aider les apprenants à utiliser des stratégies appropriées au contenu ciblés tout en 
repérant et rectifiant les concepts erronés, et le choix efficace des méthodes qui mènent à la pensée réflexive tout en ajustant ces méthodologies sur les performances et les réaction des apprenants, et aussi de les permettre à évaluer les concepts du contenu visé tout en surmontant toutes les difficultés et les obstacles qui en dérivent.

- La quatrième qui inclue la technologie, la pédagogie avec le contenu, se compose de 15 compétences concernant l'utilisation éthique du numérique afin d'encourager l'apprentissage du contenu, et le renforcement des méthodologies numériques appropriées aux différents styles d'apprentissage ; tout en guidant les étudiants à choisir l'environnement technique approprié aux objectifs visés et aussi l'intégration de la technologie avec la didactique afin de motiver les apprenants vers la formation continue pour la discipline étudiée tout en surmontant les problèmes techniques, psychiques, et épistémologiques qui réduisent l'interactivité et la créativité des apprenants.

\section{2- L'application des grilles d'évaluation :}

Afin de mesurer le degré de l'intégration du numérique dans les programmes de formation initiale des enseignants, la chercheuse s'est appuyée sur deux volets en appliquant les grilles :

- L'expérience personnelle de la chercheuse : qui, pour plus de 14 ans comme maitre de conférences au département du Curricula et de Méthodologie, s'en charge de la surveillance de l'enseignement du cursus du micro-enseignement; et aussi de l'enseignement de la méthodologie de la 3ième année, ce qui l'a mené à juger les cursus visés.

- Les rencontres accordées pour appliquer les grilles sur les cursus ciblés, avec le corps enseignant qui s'en charge de l'enseignement au département de Curricula et de méthodologie à la faculté de Jeunes Filles pour les lettres les sciences et l'éducation; ce corps enseignant se compose d'un professeur, un professeur adjoint, deux maitres de conférences, et une maitre assistante, qui ont pu (avec leurs années d'expérience qui varient entre 6 ans et 35 ans) évaluer les cursus selon le modèles TPACK.

\section{3- L'analyse des résultats de l'application des grilles d'évaluation (Annexe no2) :}

La chercheuse a calculé jusqu'à quel point les cursus de la formation des enseignants de FLE reflètent-ils les axes du modèle TPACK, elle a présenté une analyse qualitative et quantitative des résultats

\section{4-La suggestion d'un cadre de formation (Annexe no3)}

La chercheuse suggère un cadre de formation basé sur le modèle TPACK pour les enseignants dans les facultés de pédagogie afin d'intégrer au maximum le numérique dans les cursus visés, elle a précisé son importance pour les preneurs de décision, les universitaires, le corps enseignants et finalement pour les futurs enseignants qui ont les ciblés par cette recherche. 


\section{Interprétation des résultats :}

La chercheuse interprète les résultats de l'application des quatre grilles d'évaluation du modèle TPACK sur les 4 cursus de la formation initiale des enseignants dans les Faculté de Pédagogie, elle effectue une analyse qualitative et quantitative des résultats.

\section{Tableau No 1 :}

Les résultats de l'application des quatre grilles d'évaluation du modèle TPACK sur les cursus ciblés dans sa totalité

\begin{tabular}{|l|c|c|l|l|}
\hline Cursus & $\begin{array}{l}\text { Micro- } \\
\text { enseignement }\end{array}$ & $\begin{array}{l}\text { Méthodologie de } \\
\text { la 3 } 3^{\text {ième }} \text { année }\end{array}$ & $\begin{array}{l}\text { Méthodologie de } \\
\text { la 4 } \text { 4 }^{\text {ieme }} \text { année }\end{array}$ & $\begin{array}{l}\text { Stage } \\
\text { pratique }\end{array}$ \\
\hline $\begin{array}{l}\text { Le Modèle } \\
\text { TPACK }\end{array}$ & $18.5 \%$ & $32.5 \%$ & $22.5 \%$ & $14.1 \%$ \\
\hline
\end{tabular}

Ce tableau représente jusqu'à quel point les 4 Cursus de la formation initiale ont reflété le modèle TPACK dans sa totalité avec ces 4 axes. Ces résultats assurent le manque crucial des compétences numériques dans les 4 cursus par différent taux : les axes de ce modèle sont reflétés dans la fiche du stage pratique par un taux totale de $14.1 \%$, elle représente le taux le plus bas des 4 cursus de la formation initiale, tandis que le cursus de la méthodologie de la $3^{\text {ième }}$ année représente le taux le plus élevé, mais il reste toujours faible.

\section{Tableau No 2 :}

Les résultats de l'application de la grille d'évaluation concernant la connaissance pédagogique

\begin{tabular}{|l|c|c|l|c|}
\hline Cursus & $\begin{array}{l}\text { Micro- } \\
\text { enseignement }\end{array}$ & $\begin{array}{l}\text { Méthodologie de } \\
\text { la 3 } 3^{\text {ieme }} \text { année }\end{array}$ & $\begin{array}{l}\text { Méthodologie de } \\
\text { la 4 } \text { ième }_{\text {année }}\end{array}$ & $\begin{array}{l}\text { Stage } \\
\text { pratique }\end{array}$ \\
\hline $\begin{array}{l}\text { La connaissance } \\
\text { pédagogique }\end{array}$ & $25 \%$ & $25 \%$ & $25 \%$ & $30 \%$ \\
\hline
\end{tabular}

Ce tableau présente le taux de la réalisation du premier axe concernant la connaissance pédagogique ; cet axe est mis en relief dans les 4 cursus par un taux semblable; mais il demeure très faible ; ces résultats varient entre 25 et $30 \%$. Le plus élevé est celui du stage pratique, ce qui est dû à la nature de cette fiche qui s'intéresse à évaluer les performances de la connaissance pédagogique des étudiants lors de la pratique de l'enseignement dans les écoles. 


\section{Tableau No 3 :}

Les résultats de l'application de la grille d'évaluation concernant la technologie et le contenu

\begin{tabular}{|l|c|c|l|l|}
\hline Cursus & $\begin{array}{l}\text { Micro- } \\
\text { enseignement }\end{array}$ & $\begin{array}{l}\text { Méthodologie de } \\
\text { la 3 }\end{array}$ & $\begin{array}{l}\text { Méthodologie de } \\
\text { année }\end{array}$ & $\begin{array}{l}\text { Stage } \\
\text { pratique }\end{array}$ \\
\hline $\begin{array}{l}\text { Technologie et } \\
\text { contenu }\end{array}$ & $20 \%$ & $20 \%$ & $20 \%$ & $0 \%$ \\
\hline
\end{tabular}

Ce tableau reflète les résultats de l'axe concernant la technologie et le contenu. L'axe le moins représenté dans les 4 cursus ce qui assure que la formation initiale exige une réforme rigoureuse dans le domaine de l'intégration du numérique, on peut remarquer que la fiche du stage pratique, avec un taux de $0 \%$ est dépourvue de toute sorte de performance concernant l'intégration du numérique, tandis qu'elles sont représentées dans les autres cursus par un taux stable de $20 \%$ chacun.

\section{Tableau No 4 :}

Les résultats de l'application de la grille d'évaluation concernant la pédagogie et le contenu

\begin{tabular}{|l|c|c|c|c|}
\hline Cursus & $\begin{array}{l}\text { Micro- } \\
\text { enseignement }\end{array}$ & $\begin{array}{l}\text { Méthodologie de } \\
\text { la 3 }\end{array}$ & $\begin{array}{l}\text { Méthodologie de } \\
\text { année }\end{array}$ & $\begin{array}{l}\text { Stage } \\
\text { pratique }\end{array}$ \\
\hline $\begin{array}{l}\text { Pédagogie et } \\
\text { contenu }\end{array}$ & $20 \%$ & $60 \%$ & $\mathbf{3 0 \%}$ & $20 \%$ \\
\hline
\end{tabular}

Ce tableau indique les résultats de l'axe concernant la pédagogie et le contenu, le cursus de la méthodologie de la $3^{\text {ième }}$ année occupe le taux le plus élevé parmi les quatre cursus, cet axe représente l'objectif primordial que tend à réaliser les facultés de pédagogie durant la formation initiale des enseignants, mais le taux reste toujours très bas dans les 3 autres cursus, il ne dépasse pas le $20 \%$. De même la différence entre la représentation du numérique dans les deux cursus concernant la méthodologie est digne d'être étudiée, pour remédier cet écart entre eux.

\section{Tableau No 5 :}

Les résultats de l'application de la grille d'évaluation concernant la technologie la pédagogie et le contenu

\begin{tabular}{|l|c|c|l|c|}
\hline Cursus & $\begin{array}{l}\text { Micro- } \\
\text { enseignement }\end{array}$ & $\begin{array}{l}\text { Méthodologie de } \\
\text { la 3 }\end{array}$ & $\begin{array}{l}\text { Méthodologie de } \\
\text { année }\end{array}$ & $\begin{array}{l}\text { Stage } \\
\text { pratique }\end{array}$ \\
\hline $\begin{array}{l}\text { Technologie } \\
\text { pédagogie et contenu }\end{array}$ & $10 \%$ & $20 \%$ & $15 \%$ & $6.6 \%$ \\
\hline
\end{tabular}


Les résultats de ce tableau révèlent l'insuffisance du cloisonnement des performances technologiques avec les autres composantes du modèle TPACK, ce qui était observé le plus dans la fiche du stage pratique qui reflète ces compétences par un taux de $6.6 \%$, ce qui assure l'absence quasi-complète des TICE, et qui exige aussi une réforme primordiale dans le statut de cette fiche d'évaluation. De même le taux que représente le cursus du micro-enseignement ne dépasse pas le $10 \%$ des compétences technologiques, tandis que les 2 autres cursus sont reflétés par un taux de $20 \%$. Ce qui sollicite une collaboration entre les responsables; les universitaires et les chercheurs dans le domaine afin de mener à bien des recherches qui incitent l'intégration du numérique avec toutes ses dimensions dans les cursus universitaires.

\section{Tableau No 6}

Dans les tableaux suivants, la chercheuse va présenter les résultats de l'analyse de chaque cursus à part, en esquissant le taux de représentation de chaque axe du modèle TPACK afin de présenter une vision globale qui aide à déterminer les points faibles et de pouvoir en remédier

\section{Le cursus du micro-enseignement et le modèle TPACK}

\begin{tabular}{|l|l|l|l|l|l|}
\hline $\begin{array}{l}\text { Modèle } \\
\text { TPACK }\end{array}$ & $\begin{array}{l}\text { La connaissance } \\
\text { pédagogique }\end{array}$ & $\begin{array}{l}\text { Technologie } \\
\text { et contenu }\end{array}$ & $\begin{array}{l}\text { Pédagogie } \\
\text { et contenu }\end{array}$ & $\begin{array}{l}\text { Technologie, pédagogie } \\
\text { et contenu }\end{array}$ & Totale \\
\hline $\begin{array}{l}\text { Micro- } \\
\text { enseignement }\end{array}$ & $25 \%$ & $20 \%$ & $20 \%$ & $10 \%$ & $18.5 \%$ \\
\hline
\end{tabular}

Pour le cursus du micro-enseignement : les résultats indiquent que les performances du modèle TPACK sont reflétés dans le cursus du micro-enseignement par un taux très faible $(18.5 \%)$.

On remarque que le premier axe concernant la connaissance pédagogique est le plus représenté par un taux de $25 \%$, par suite les deux axes de la technologie avec le contenu et l'axe de la pédagogie et le contenu occupent $20 \%$, tandis que l'axe concernant la technologie est presque absent, il est reflété par un taux très qui ne dépasse pas le 10\%. Ce qui exige une réforme complète dans la description de ce cursus avec la nécessité d'une intégration optimale du numérique.

\section{Tableau No 7}

Le cursus de la méthodologie de la $3^{\text {ième }}$ année et le modèle TPACK

\begin{tabular}{|l|l|l|l|l|l|}
\hline Modèle TPACK & $\begin{array}{l}\text { La connaissance } \\
\text { pédagogique }\end{array}$ & $\begin{array}{l}\text { Technologie } \\
\text { et contenu }\end{array}$ & $\begin{array}{l}\text { Pédagogie } \\
\text { et contenu }\end{array}$ & $\begin{array}{l}\text { Technologie, } \\
\text { pédagogie et } \\
\text { contenu }\end{array}$ & Totale \\
\hline $\begin{array}{l}\text { La méthodologie } \\
\text { de la 3ièmé année }\end{array}$ & $25 \%$ & $20 \%$ & $60 \%$ & $20 \%$ & $32.5 \%$ \\
\hline
\end{tabular}


En ce qui concerne le cursus de la méthodologie de la $3^{\text {ième }}$ année $:$ Ce tableau nous révèle les résultats de l'application du modèle TPACK. Ce cursus occupe le taux le plus élevé parmi les cursus évalués avec un pourcentage de $32.5 \%$; l'axe le plus reflété est celui de la pédagogie et le contenu tandis que les trois autres axes sont traduits par un taux qui varie entre le 20 et le $25 \%$, ces résultats demeurent aussi très insatisfaisants, et exigent des efforts des universitaires et des formateurs et des preneurs de décision afin d'intégrer au maximum le numérique dans la formation des enseignants.

\section{Tableau No 8}

Le cursus de la méthodologie de la $4^{\text {ième }}$ année et le modèle TPACK

\begin{tabular}{|l|l|l|l|l|l|}
\hline Modèle TPACK & $\begin{array}{l}\text { La connaissance } \\
\text { pédagogique }\end{array}$ & $\begin{array}{l}\text { Technologie } \\
\text { et contenu }\end{array}$ & $\begin{array}{l}\text { Pédagogie } \\
\text { et contenu }\end{array}$ & $\begin{array}{l}\text { Technologie, } \\
\text { pédagogie et } \\
\text { contenu }\end{array}$ & Totale \\
\hline $\begin{array}{l}\text { La méthodologie } \\
\text { de la 4 4ème année }\end{array}$ & $25 \%$ & $20 \%$ & $30 \%$ & $15 \%$ & $22.5 \%$ \\
\hline
\end{tabular}

Pour le cursus de la méthodologie de la $4^{\text {ième }}$ année, ce tableau prouve que le cursus concernant la méthodologie de la $4{ }^{\text {ième }}$ année est dépourvue de toute intégration du numérique même sur le niveau théorique ce qui exige une reformulation des thèmes traitées pour pouvoir intégrer le numérique d'une manière efficace, adoptée et appropriée.

\section{Tableau No 9}

\section{La fiche du stage pratique et le modèle TPACK}

\begin{tabular}{|l|l|l|l|l|l|}
\hline $\begin{array}{l}\text { Modèle } \\
\text { TPACK }\end{array}$ & $\begin{array}{l}\text { La connaissance } \\
\text { pédagogique }\end{array}$ & $\begin{array}{l}\text { Technologie } \\
\text { et contenu }\end{array}$ & $\begin{array}{l}\text { Pédagogie } \\
\text { et contenu }\end{array}$ & $\begin{array}{l}\text { Technologie, } \\
\text { pédagogie et } \\
\text { contenu }\end{array}$ & Totale \\
\hline $\begin{array}{l}\text { La fiche du } \\
\text { stage pratique }\end{array}$ & $30 \%$ & $0 \%$ & $20 \%$ & $6.6 \%$ & $14.1 \%$ \\
\hline
\end{tabular}

On peut remarquer que la fiche du stage pratique exige plus d'efforts dans son élaboration, afin de refléter les performances que doit posséder les enseignants, en ce qui concerne les connaissances pédagogiques et aussi le cloisonnement entre la technologie, la pédagogie et le contenu, afin de pouvoir former un enseignant qui répond aux exigences de la nouvelle technologique et le niveau mondial de l'application et l'intégration du numérique. 


\section{Tableau No 10 : Les 4 cursus et le modèle TPACK}

Afin de comparer les résultats des 4 cursus, la chercheuse les a présentés dans ce tableau :

\begin{tabular}{|l|c|c|c|c|}
\hline \multicolumn{1}{|c|}{ Modèle TPACK } & $\begin{array}{l}\text { Micro- } \\
\text { enseignement }\end{array}$ & $\begin{array}{l}\text { Méthodologie de } \\
\text { la } \mathbf{3}^{\text {ième }} \text { année }\end{array}$ & $\begin{array}{l}\text { Méthodologie de } \\
\text { la 4ième } \\
\text { année }\end{array}$ & $\begin{array}{l}\text { Stage } \\
\text { pratique }\end{array}$ \\
\hline Connaissance pédagogique & $25 \%$ & $25 \%$ & $25 \%$ & $30 \%$ \\
\hline Technologie et contenu & $20 \%$ & $20 \%$ & $20 \%$ & $0 \%$ \\
\hline Pédagogie et contenu & $20 \%$ & $60 \%$ & $\mathbf{3 0 \%}$ & $20 \%$ \\
\hline $\begin{array}{l}\text { Technologie, pédagogie et } \\
\text { contenu }\end{array}$ & $10 \%$ & $20 \%$ & $15 \%$ & $6.6 \%$ \\
\hline Totale & $75 \%$ & $125 \%$ & $90 \%$ & $56.6 \%$ \\
\hline La moyenne & $18.5 \%$ & $32.5 \%$ & $22.5 \%$ & $14.1 \%$ \\
\hline
\end{tabular}

La chercheuse a calculé le pourcentage de la représentation des items de chaque axe à part pour chaque cursus, par suite elle a additionné le taux des 4 axes et l'a divisé sur 4 pour déterminer la moyenne de présentation de chaque cursus ; un regard attentif sur ces résultats, assure la manque cruciale, l'absence et la négligence presque complète du numérique et de ses outils dans la description et la pratique des cursus de la formation initiale à la faculté de Jeunes filles section de la langue française pédagogie.

\section{Discussion des résultats :}

Les résultats de la recherche ont montré que malgré la disponibilité de certain matériel TICE à la portée des apprenants et de leurs enseignants, l'usage des TICE dans les pratiques des enseignants reste très limité, voire absent. En réalité, l'analyse de ces résultats a permis l'identification de quelques obstacles à étudier :

- Les obstacles relatifs à l'infrastructure des TICE au niveau des établissements scolaires et universitaires : malgré l'énorme investissement de ressources financières et humaines ainsi que l'offre de la formation, celle-ci reste insuffisante et ne fournit pas aux enseignants les compétences nécessaires leur permettant d'utiliser efficacement les TICE dans leurs pratiques d'enseignement.

- Les obstacles relatifs au développement professionnel en matière des TICE : Il est légitime de s’interroger sur la réalité du développement des compétences des enseignants, en matière des TICE, et de leur appropriation des techniques de base leur permettant l'intégration de ces technologies dans leurs pratiques enseignantes.

En d'autres termes, les universitaires possèdent-ils les compétences et les connaissances de base en informatique? Quel est leur degré de maîtrise des logiciels multimédias 
permettant la création ou la réadaptation des ressources numériques ? Ces enseignants possèdent-ils les capacités à faire usage des TICE dans leurs enseignements ?

En effet, le manque de compétences TICE des enseignants était un obstacle majeur entravant l'usage des TICE dans leurs enseignements, aussi, le manque des compétences TICE constitue la raison principale qui a poussé les enseignants à ne pas utiliser ces technologies dans l'enseignement.

Pour conclure, on peut assurer que la formation des enseignants demeure le pivot de n'importe quelle reforme concernant l'intégration des TICE dans le domaine de l'enseignement, elle reste le point déclencheur de toute évolution des programmes et des cursus universitaires, ce qui exige l'élaboration des stages de formations qui leur permettre d'acquérir les compétences nécessaires pour utiliser les TICE en contexte scolaire, de façon professionnelle et durable et non pas uniquement le développement de qualifications en alphabétisme informatique.

\section{Cadre de formation suggérée (Annexe no 3 :)}

Ce cadre de formation proposé préconise la vision d'une intégration efficace et d'une exploitation optimale du numérique au service de la réussite des futurs enseignants, qui leur permettent de développer et de maintenir leurs compétences tout au long de leur vie professionnelle. Ainsi, ce cadre de formation générale et professionnelle de la compétence numérique regroupe les dimensions jugées indispensables pour apprendre et évoluer au 21e siècle; on y préconise des différentes variables qui entrent en jeu dans l'intégration des TICE citant ainsi l'institution, les enseignants, les apprenants, les logiciels disponibles et le dispositif spatial humain.

La chercheuse abordera ainsi le rôle de chacun de ses éléments :

En effet, l'institution, représenté ici par les Facultés de Pédagogie, inclura le programme de formation qu'on prétend évoluer lors de l'application de la recherche. Les enseignants incluront les professeurs d'universités et les éducateurs avec lesquels les futursenseignants vont collaborer. Les apprenants incluront les enseignants stagiaires et leurs étudiants durant les stages pratiques; et finalement, les logiciels incluront les logiciels disponibles au sein du programme de formation dans les écoles et les lycées où les futursenseignants seront placés. Chacune de ces variables a des caractéristiques qui vont intervenir dans l'intégration des TICE et dans les changements pédagogiques qui en découleront.

Cette recherche illustre de façon détaillée la façon dont ces variables entrent en jeu et interagissent entre elles dans un tel cadre, afin de pouvoir intégrer le numérique dans le cadre de formation initiale des futurs enseignants dans les facultés de pédagogie en se basant sur le modèle TPACK.

En ce qui concerne les Facultés de pédagogie, elles doivent fournir certaines contraintes spatiotemporelles telles que la distribution des salles et des horaires, et la séquence pédagogique créée au sein du programme de formation doit être destinée à un cadre idéal où l'Internet est facile 
d'accès et où les TICE sont nombreuses et récentes; de même les futurs-enseignants doivent prendre conscience de la différence entre le cadre idéal et la réalité vécue, certains ont dû apprendre à intégrer les TICE lorsqu'il n'y avait qu'un ordinateur pour tous les étudiants, ou lorsque les pages web qu'ils voulaient utiliser n'étaient pas accessibles à cause des filtres.

On doit aussi mettre en considération que chaque institution pratique sa politique concernant l'intégration des TICE, traduite dans les règles d'accès à l'ordinateur, la qualité et la quantité des TICE disponibles. De ce fait, les enseignants stagiaires doivent développer les compétences qui leur permettent de modifier les connaissances acquises au sein du programme de formation, et de les adapter aux différents contextes.

En ce qui concerne les universitaires et le corps professoral, ils doivent être bien formés sur le niveau numérique afin de pouvoir développer ces compétences chez leurs apprenants, de même ils doivent se baser dans la conception de leurs modules enseignés sur les référentiels TICE, ces référentiels qui représentent le fruit de tant d'études, des recherches et des projets élaborés par l'union européen et d'autres pays comme le Canada et les états unis.

Ces référentiels ont un point commun qui réside dans leur participation à la mise en place des normes permettant de s'interroger continuellement sur les besoins réels en matière de compétences requises pour l'usage et l'intégration efficace de ces technologies en éducation.

De même, le corps professoral dans les facultés de pédagogie doit être expert dans les contenus enseignés et dans la méthodologie de leur transmission comme de celle de la construction des savoirs par les étudiants, s'est approprié l'épistémologie, les contenus, les concepts et les démarches de ses disciplines ou spécialités. Il est à même de concevoir, de conduire, de réguler et d'évaluer des situations d'apprentissage riches de sens et de savoir pour tous les étudiants, ce qui suscite leur envie d'apprendre, de réfléchir et de progresser, et contribue à développer leur esprit critique. Utilisant à bon escient les outils et les ressources pédagogiques, et les outils numériques, leur propose des supports d'apprentissage de qualité et leur mènent à réaliser le plaisir d'apprendre et d'apprendre avec plaisir.

En ce qui concerne les futurs enseignants : Au début de leur apprentissage, on leur propose de passer par des cours de préparation pour se familiariser avec les logiciels tels que le Word, l'Excel, qui pouvaient être utilisés pour créer facilement des leçons illustrées et interactives, pour calculer les notes, pour stocker un grand nombre d'informations.

Aussi de les aider à acquérir les habilités technologiques, la création de sites Web, et la numérisation d'images utiles à l'enseignement du FLE; de les encourager à acquérir les compétences liées à l'agencement de site web tels que (le format de page, l'utilisation de couleurs, d'images, et le typographie) ; de les entrainer à créer un portfolio numérique qui incluait entre autres la création d'un scénario pédagogique entièrement fondé sur Internet. Ce scénario pouvait être utilisé à distance ou en présentiel. Afin de créer ce portfolio, les futurs-enseignants devaient se familiariser avec des logiciels tels que (le FrontPage et Powtoon.) Si au départ on se trouve 
dans le cas où ils n'étaient pas enthousiastes en ce qui concerne l'intégration des TICE, la diversité des sites où ils se trouvaient pouvait renforcer leurs attitudes.

Ces futurs-enseignants doivent au cours de leur départ de formation, travailler en collaboration avec une assistante ou une maitre-assistante qui les encadrait. Ils doivent avoir accès à toutes les ressources humaines (techniciens, collègues, etc.), matérielles (casiers, classeurs, etc.), et technologiques (photocopieuses, salles multimédia, logiciels, etc.) disponibles dans leurs Facultés. Ils doivent développer leurs autonomies lorsqu'ils utilisent le numérique dans un contexte pédagogique ou professionnel ou encore dans la vie quotidienne. Cette autonomie doit leurs permettre de choisir judicieusement les outils numériques à utiliser lorsqu'ils doivent accomplir une tâche particulière.

Enfin, la compétence numérique est intimement liée au développement professionnel de tous les travailleurs du 21e siècle. En ce sens, il est nécessaire que tous soient en mesure d'utiliser les ressources numériques disponibles, telles que les communautés de pratique, les formations en ligne ou les tutoriels, pour maintenir leurs compétences professionnelles à jour.

En ce qui concerne les logiciels TICE : il faut intégrer des logiciels qui permettaient de pratiquer :

- La grammaire de manière ludique, ce genre d'activités attirait l'attention des étudiants. De plus, grâce à ce genre d'activités les enseignants stagiaires pouvaient mieux gérer le comportement des étudiants indisciplinés :

- Le vocabulaire : un cours de vocabulaire avec les matériels numériques rend d'abord le travail des professeurs des langues plus facile. Les matériels numériques permettent la manipulation de l'information d'une manière plus efficace. Les professeurs peuvent préparer les cours et exploiter les différents canaux de communication comme le texte, l'audio, l'image dans une même projection; ils gagnent de cette façon non seulement du temps mais l'attention des étudiants avec des mis en situation authentique et didactique.

- La phonétique et la prononciation : Un professeur sans tous les moyens technologiques prendra du temps à préparer des fiches à la main, prendra du temps pour prononcer lui-même en cours et écrire à chaque fois les transcriptions des mots. Se mettre dans un environnement numérique rend l'apprentissage du FLE plus motivant pour les étudiants parce qu'ils peuvent pratiquer la langue même en jouant.

- Les jeux éducatifs : Dans un cours sans accès à l'ordinateur, le professeur peut créer un jeu mais le résultat sera plus long: d'abord parce que l'étudiant doit attendre à chaque fois son tour au lieu de pratiquer tous en simultané. De même les nouvelles générations avec l'explosion des TICE apprennent plus rapidement et se fatiguent aussi facilement avec des activités monotones.

Malgré les grands efforts que font la plupart des enseignants pour transmettre les connaissances aux étudiants dans l'éducation publique, le gouvernement doit assurer des 
établissements bien équipé avec des matériaux numériques non seulement pour faciliter la tâche des enseignants mais aussi pour rendre l'éducation plus agréable.

En ce qui concerne les compétences qu'on tend à développer lors de l'enseignement des cursus de la formation initiale (Annexe no3) et qui contribuent au développement des compétences technopédagogiques de nos futurs-enseignants afin d'intégrer le numérique en se basant sur le modèle TPACK, on doit attirer l'attention des concepteurs des cursus à deux grandes categories de compétences :

- La première concerne les compétences générales relatives à l'exercice du métier d'enseignement et qui comprend : la " maîtrise de l'environnement numérique professionnel », « le développement des compétences pour la formation tout au long de la vie » et « la responsabilité professionnelle dans le cadre du système éducatif ». L'acquisition de cette première catégorie de compétences devra également permettre aux enseignants d'utiliser les TICE dans leurs tâches professionnelles, par exemple dans la recherche de ressources via Internet pour améliorer leurs cours.

- La deuxième catégorie concerne l'ensemble des compétences permettant l'intégration efficace des TICE dans l'enseignement et qui comprend les compétences relatives à la capacité d'utilisation des outils permettant le travail collaboratif et en réseau, les compétences relatives à la « conception et la préparation de contenus d'enseignement et de situations d'apprentissage», les compétences relatives à la capacité de mettre en œuvre l'usage pédagogique des TICE dans des situations d'apprentissage et les compétences relatives à la mise en œuvre des TICE dans l'évaluation des apprentissages.

On doit leur faire apprendre les compétences de base en matière des TICE, à savoir l'acquisition d'une culture numérique, ainsi que les moyens suffisants permettant aux enseignants de faire le meilleur choix et le meilleur usage de logiciels et de contenus numériques appropriés pour des situations d'apprentissage en salle de classe, en salle multimédia ou en salle d'informatique.

L'acquisition de cette catégorie de compétences doit suivre l'approche relative à l'approfondissement des connaissances des enseignants et comporte la capacité de gestion de l'information et d'intégration des outils logiciels propres à une discipline d'enseignement, dans une approche pédagogique centrée sur l'étudiant, afin de leur permettre d'acquérir les compétences nécessaires à la résolution des problèmes plus complexes. Cette approche vise également l'acquisition de compétences permettant aux enseignants de travailler en réseau afin de pouvoir aider les étudiants dans la réalisation de leurs projets collaboratifs menés en ligne.

- Aussi on doit se focaliser sur la création des connaissances et qui vise à l'acquisition par les enseignants des compétences qui leur permettent de concevoir des ressources numériques d'apprentissage, d'aider les étudiants, en utilisant les TICE, et aussi à acquérir les compétences nécessaires au travail collaboratif, à la création de nouvelles compétences et à exercer leur esprit critique. 
Pour conclura on doit assurer sur le fait que les enseignants doivent être capables de structurer et de concevoir des situations d'apprentissage dans lesquelles les étudiants peuvent appliquer les compétences numériques. Par conséquent, la classe et par suite l'établissement deviennent une communauté d'apprentissage fondée sur l'innovation, la formation et la création de nouvelles connaissances basées sur les TICE.

\section{Suggestion et recommandation :}

A la fin de cette recherche, on peut y présenter les suggestions et les recommandations suivantes :

- Préparer des activités enrichissantes pour les cursus de formation initiale basés sur le modèle TPACK.

- Utiliser le modèle TPACK pour évoluer les méthodes d'enseignement de FLE au cycle pré-universitaire ; soit le cycle secondaire ou le cycle préparatoire.

- Se servir du modèle TPACK pour enrichir les cursus de l'enseignement de FLE dans la filière technique de l'enseignement soit commerciale, agriculture, industrielle et touristique.

- Préparer des stages de formation des enseignants universitaires et enseignants du FLE pour les préparer à l'intégration du numériques dans leur vie professionnelle.

- Formuler des MOOC ou CLOM (classe en ligne ouverte et massive) pour les enseignants qui ne peuvent pas se déplacer pour assister à des stages de formation.

- Intégrer la formation hybride dans l'enseignement universitaire afin de faciliter la communication et l'échange entre apprenants et enseignants et de compenser le temps et les matériels couteux.

- Inciter les universités et les centres de recherche à produire des ressources et de mener des recherches en pédagogie et didactique afin d'intégrer les TICE dans notre système éducatif.

- Exploiter les ressources locales, dans la mesure du possible pour garantir le plus haut degré possible de participation et d'investissement.

\section{Conclusion}

Bref, ce cadre de formation vise à favoriser le développement de la compétence numérique dans l'ensemble de la communauté éducative pour que les futurs enseignants des facultés de pédagogie soient des praticiens réflexifs et autonomes, dans leur utilisation du numérique. Cette habileté est primordiale dans notre contexte est marqué par des innovations technologiques qui transforment le marché de l'emploi et influent sur les compétences recherchées par les employeurs. En ce sens, on a essayé d'adapter la pratique enseignante et d'inclure les compétences numériques dans les cursus de la formation initiale pour préparer les futurs enseignants aux défis de demain. 


\section{Bibliographie :}

- Bangou, Francis. (2013) Cartographies of Becoming in Education. DOI.

- Becta (2009-2010). Harnessing Technology Work-based Learning Sector.

- Brodin, É. (2002). "Innovation, instrumentation technologique de l'apprentissage des langues: des schèmes d'action aux modèles de pratiques émergentes". Apprentissage des Langues, Systèmes d'Information et de Communication (Alsic), vol. 5, n 2. pp. 149-181.

- Fastrez Pierre, De Smedt Thierry (2013). Les compétences en Littératie médiatique. De la définition aux nouveaux enjeux éducatifs. Dans Medialoc, Vol. 1, no 11, p 2-8.

- Faten Abdel Meguid El Seoudy (2017). Évoluer les programmes du développement professionnelle des enseignants des sciences commerciales. Faculté de Pédagogie. Université de Tanta.

- Francis Bangou, (2006). «Intégration des TICE et apprentissage de l'enseignement : une approche systémique», Alsic Vol. 9 .

- Freeman, D. \& Johnson, K. (1998). "Reconceptualizing the Knowledge-Base of Language Teacher Education". TESOL Quarterly, n 32. pp. 397-416.

- Gerbault, J. (2002). "Technologies de l'Information et de la Communication et diffusion $\mathrm{du}$ français - Usages, représentations, politiques". Apprentissage des Langues, Systèmes d'Information et de Communication (Alsic), vol. 5, $\mathrm{n}^{\circ}$ 2. pp. 183-207.

- Johnson, L., Adams Becker, S., Estrada, V. et Freeman, A. (2015). The NMC Horizon Report: 2015 K-12 Edition.

- Kabbas, Azza: (2017) «Modèle TPACK l'un des modèles contemporains pour déterminer et évaluer les caractéristiques de l'enseignant efficace de la XXIème siècle. XVIIIème Séminaire de l'encadrement pédagogique (l'enseignant du 2030) Le Royaume de l'Arabie Saoudite ; Zone Limitrophe du Nord.

- Koehler, M. J., \& Mishra, P. (2009). What is technological pedagogical content knowledge? Contemporary Issues in Technology and Teacher Education, 9(1), 60-70.

- Levin, B.B. \& Ammon, P.A. (1996). "A Longitudinal Study of the Development of Teachers' Pedagogical Conceptions : The Case of Ron". Teacher Education Quarterly, vol. 23. pp. 5-25.

- Mangenot, F. (2000). "L'intégration des TIC dans une perspective systémique". Les Langues Modernes, $\mathrm{n}^{\circ}$ 3. pp. 38-44.

- (Lefebre.2014) traduction en français du Modèle TPACK (Koehler et Mishra. 2009) Le schéma du modèle TPACK.

- Petitgirard, J.-Y. (2004). "Introduction". Les Lettres Modernes, n 4. pp. 7-13. 
- SHULMAN L. S. (2004). The Wisdom of Practice: Essays onTeaching, Learning, and Learning to Teach. San Francisco : Jossey-Bass Publishers.

- Shulman, L.S. (2007). Ceux qui comprennent :Le développement de la connaissance dans l'enseignement. Education et Didactique, 1(1), 97-114.

- Trésor de la langue française dictionnaire (2004) publié en 1994 et Format CDrom Gallimard.

- Van Lier L. (1999). «Une perspective écologique », in Caré J.-M. (dir.) Le Français dans le monde, Recherches et applications, Apprendre les langues étrangères autrement (janvier 1999), p. 10-20. Paris, Hachette-Edicef.

- Wertsch, J. (1981). The concept of activity in Soviet Psychology: An introduction. Armonk : Sharpe.

\section{Sitographie :}

- http://alsic.u-strasbg.fr/Num09/brodin/alsic_n09-rec3.htm

- http://alsic.u-strasbg.fr/Num09/gerbault/alsic_n09-pra1.htm

- http://journals.openedition.org/alsic/290

- https://www.youtube.com/watch?v=IODpULBMBpI

- https://www.youtube.com/watch?v=RPopXNhgo_A

- $\quad$ https://tecfalabs.unige.ch/mitic/articles/koehler_mishra_2009_what_is_technological_ped agogical_content_knowledge.pdf

- $\quad$ http://cdn.nmc.org/media/2015-nmc-horizon-report-k12-EN.pdf

- $\quad$ http://dera.ioe.ac.uk/1685/1/becta_2010_wblsurvey_report.pdf 


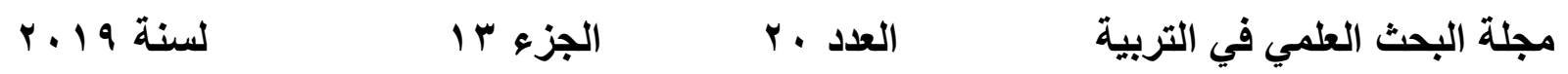

\title{
في ضوء نموذج دراسة تحليليه لبرنامج اعداد معلمي اللغة الفرنسية
}

\author{
د. نسرين صلاح عبد الغني \\ قسم المناهج وطرق التّريس كليه البنات جامعه عين شمس \\ Nesrine.salah@womein.asu.edu.eg
}

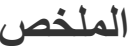

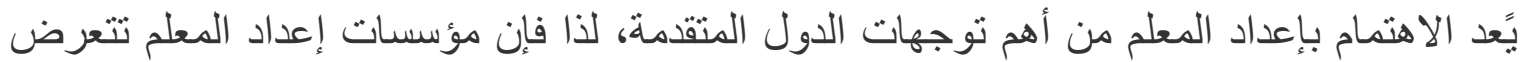

للنقد و التقويم، و في ضوء المستجدات الحديثة في مجال التربية وخاصه في مجال التكنولوجي، ظهرت التهاعن العديد

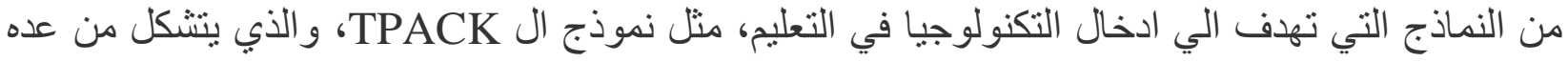
ابعاد ثتداخل بعضها مع البعض، حيث يشتمل علي ثنلاثة ابعاد: البعد الأول والخاص بالمعرفة فئة بالمحتوي

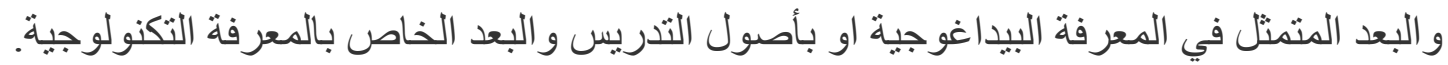
وفي دراسة تحليليه لمقررات اعداد المعلم و المعني بتدريسها قسم المناهج وطرق التدريس بكليات

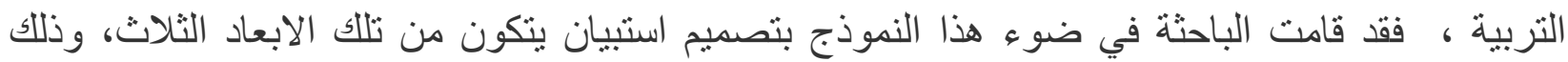
للوقوف علي مدي ما تضمنه مقررات التدريس المصغر وطرق تدريس اللغة الفرنسية في العامين الثالث و الر ابع وكذلك أدوات التربية العملية سواء المنفصلة منها او المتصلة، من مهار ات تكنولوجيه تساهم في تلئي التنمية المستدامة للمعلم بعد تخرجه بما تتيحه له من برامج ، وبعد اطلاع الباحثة علي تللك المقررات وبتحليل نتائج اللقاءعات التي اجرتها مع أعضاء هيئه التدريس والهيئة المعاونة بكليه البنات، فقد اثتتت نلأك النتائج القصور الثديد فيما يخص ادماج المهار ات التكنولوجية وتوظيفها في المقررات الجامعية ، مما حدي بالباحثة

الي تقديم اطار تدريبي مقترح لتطوير تللك المقرر ات في ضوء نموذج ال TPACK

الكلمات المفتاحية : برامج اعد/د المعلم ، ندوذج ال TPACK ، دمج التكنولوجيا في التدربي ، بر/مج اعد/د معلم اللغة الفرنسية 